\title{
Testere Dişi Çift Bariyer Yapısında Rezonans Tünelleme Olayının İncelenmesi
}

\author{
Mehmet BATI \\ Recep Tayyip Erdoğan Üniversitesi, Fen Edebiyat Fakültesi, Fizik Bölümü
}

\section{$\ddot{O} z$}

Elektrik alan öngerilimi altındaki testere dişi çift bariyer yapısında rezonans tünelleme özelliği, sonlu farklar metodu bazlı denge-dışı Green fonksiyonu yöntemiyle incelenmiştir. Elektrik alan altında testere dişi çift bariyer yapılarının tünelleme iletim olasılığı ve rezonans enerji durumları sunulmuştur. Nümerik sonuçlar, iletim katsayısındaki rezonans pikinin, öngerilim alanına ve yapı parametrelerine kuvvetli bir şekilde bağlı olduğunu göstermektedir.

Anahtar Kelimeler: Denge-dışı Green fonksiyonu, sonlu farklar yöntemi, testere dişi çift bariyer, rezonans tünelleme

\section{Investigation of Resonant Tunneling Properties of Sawtooth Double Barrier Structures}

\begin{abstract}
Resonant tunneling properties of sawtooth double barrier structures under the electric field bias are investigated in this paper using the non-equilibrium Green's functions method based on finite difference method. Tunneling transmission probability and resonant energy states of sawtooth double barrier structures under the electric field are presented. Numerical results reveal that the resonant peak in the transmission coefficient depends strongly on the bias field and structure parameters.
\end{abstract}

Keywords: Non-equilibrium Green's function, finite difference method, sawtooth double barrier, resonant tunneling.

\section{Giriş}

Rezonant tünelleme olayının keşfi ile [1] düşük güç tüketen, yüksek anahtarlama hızına sahip ( $\mathrm{THz}$ mertebesinde) diyotlar üretilebilmektedir [2]. Teknolojik olarak birçok uygulama alanına sahip olan rezonans tünelleme aygıtları üzerine teorik ve teknolojik açıdan çok sayıda çalışma yapılmaktadır [3-5]. Rezonans tünelleme olayının incelenmesi için, Wigner fonksiyonu yöntemi [6-7], Monte Carlo yöntemi [8-9] transfer matris yöntemi [10] ve denge-diş1 Green fonksiyonu (NEGF) [11-12] yöntemi gibi simülasyon teknikleri mevcuttur.

Nano ölçekli aygıt üretim teknolojisinin son yıllardaki gelişimi sayesinde yüksek kaliteli farklı geometrilerde (dikdörtgen, parabolik, üçgen vb) rezonant tünelleme yapıları üretilebilmektedir [13]. Dikdörtgen çift bariyer yapısının basit olması farklı yöntemleri test etme amacıyla da kullanılmış olması nedeniyle üzerinde çok sayıda çalışma yapılmıştır $[3,14]$. Farklı geometriye sahip yapılarda elektron taşınımını inceleyen çalışmalar mevcuttur. Ters parabolik çift bariyer yapısında elektronik taşınımı Batı ve çalışma arkadaşları tarafından incelenmiştir [15]. Ohmukai üçgen çift bariyer yapısında rezonans tünelleme olayının yapı parametresine bağlılı̆̆ını transfer matrisi yöntemi ile incelemiştir [16]. Ayrıca Wang ve arkadaşları bu yapıyı elektrik alan altında yine transfer matrisi yöntemiyle incelemiştir [17]. Bu yapıda iletkenlik hesabı Luo tarafından hesaplanmıştır [18].

$\mathrm{Bu}$ çalışmada TDÇB yapısının rezonans tünelleme karakteristiği yapı parametreleri (kuyu genişliği, bariyer kalınlığı ve yüksekliği) ve elektrik alan öngerilimine bağlılığı incelenecektir. Yapının tanıtımı ve kullanılan yöntem 2. Bölümde anlatılacak olup, 3. Bölümde elde edilen veriler yorumlanacak, sonuçlar 4. Bölümde sunulacaktır.

\section{Model ve Yöntem}

Testere dişi çift bariyer potansiyelinin şematik gösterimi Şekil-1 deki gibidir. $V_{L}$ ve $V_{R}$ sırasıyla sol ve sağ potansiyel bariyer yüksekliğini, $W_{L}$ ve $W_{R}$ 
sırasıyla sol ve sağ potansiyel bariyer genişliklerini ve $L_{W}$ iki bariyer arasında kalan kuyu genişliğini göstermektedir Her bir bölge sınırı konumları $L_{i}$ $(i=1,2,3,4)$ ile belirtilmektedir.

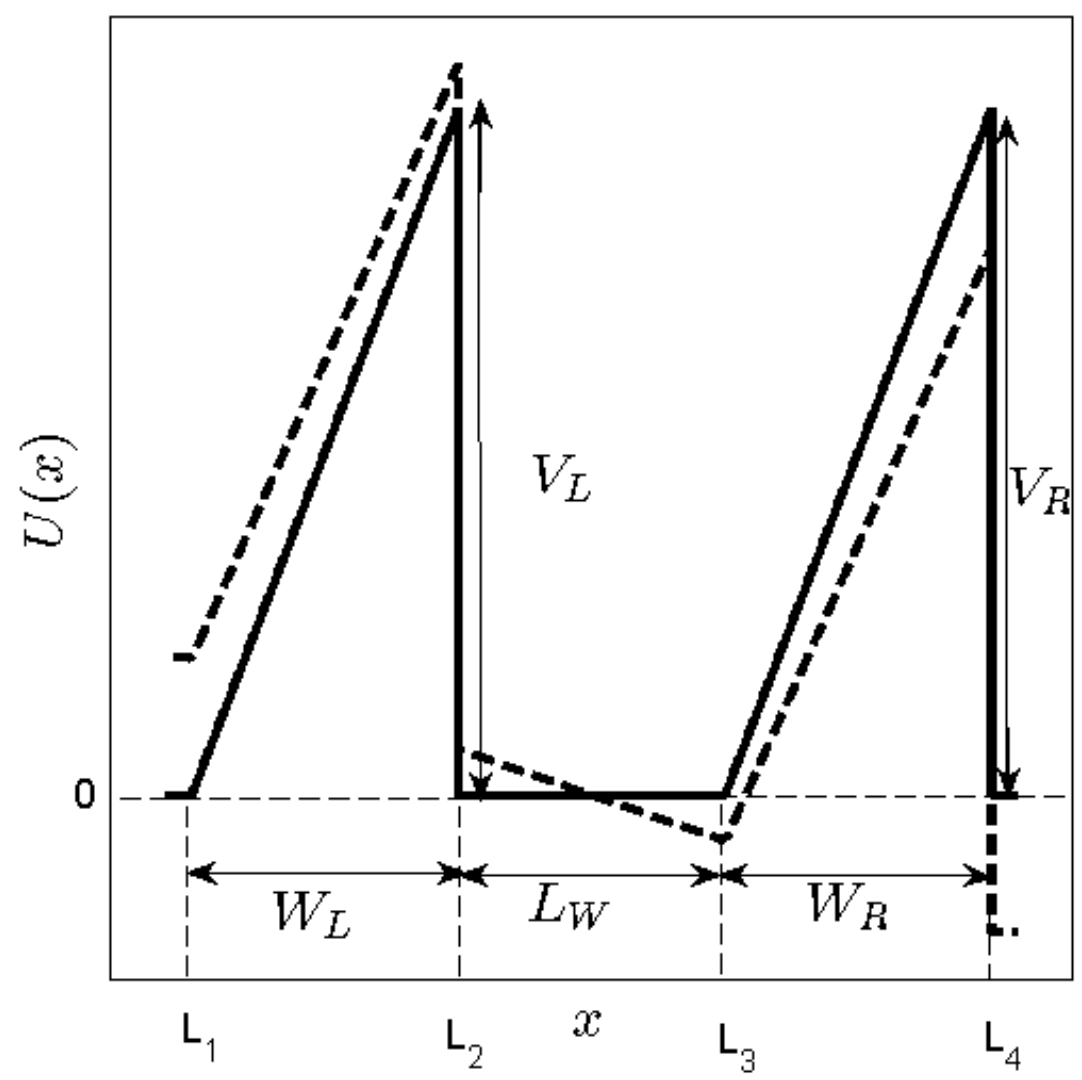

Şekil 1 Testere-dişi çift bariyer potansiyeli yapısı, sürekli çizgi elektrik alan biası yokluğundaki, kesikli çizgi elektrik alan biası varlığındaki yapıyı göstermektedir. (Sawtooth double barrier potential structure, the solid line represents the potential profile without electric field bias and dashed lines stand for case when the electric field is applied.)

Sonlu farklar yöntemi bazlı denge-dışı Green fonksiyonu (SFY-DDGF) yöntemi kullanılacaktır. Öncelikle sisteme ait bir boyutlu Schrödinger dalga denklemi

$$
-\frac{\hbar^{2}}{2 m^{*}} \frac{d^{2} \psi}{d x^{2}}+U(x) \psi=E \psi
$$

tanımlanmalıdır. Burada, $E$ elektron enerjisi ve $m^{*}$ elektronun etkin kütlesidir.

Elektrik alan $(F)$ biası altındaki TDÇB yapısının potansiyel enerji profilinin fonksiyonel formu

$$
\left\{\begin{array}{cr}
-e F L_{1}, & x(x)= \\
-\left(e F+\frac{V_{L}}{W_{L}}\right) x+\left(\frac{L_{2}}{W_{L}}+1\right) V_{L}, & L_{1} \leq x \leq L_{2} \\
-e F x, & L_{2}<x<L_{3} \\
-\left(e F+\frac{V_{R}}{W_{R}}\right) x+\left(\frac{L_{4}}{W_{R}}+1\right) V_{R}, & L_{3} \leq x \leq L_{4} \\
-e F L_{4}, & L_{4}<x
\end{array}\right.
$$

şeklindedir.

Çalışmamızda nümerik olarak hesapların daha kolay hesaplanabilmesi için boyutsuz parametrelerle çalıșacağız. Uzunluklar etkin Bohr yarıçapı $\left(a_{0}^{*}=4 \pi \varepsilon_{0} \varepsilon_{r} \hbar^{2} / m^{*} e^{2}\right)$, enerjiler etkin Hartree enerjisi $\left(E_{H}^{*}=\hbar^{2} / m^{*} a_{0}^{*}\right)$ cinsinden, ölçeklendirilecektir. Böylelikle ölçeklendirilmiș Schrödinger dalga denklemi boyutsuz nicelikler $\tilde{x}$ ve $\widetilde{U}$ cinsinden

$$
-\frac{1}{2} \frac{d^{2} \psi}{d \tilde{x}^{2}}+\widetilde{U}(\tilde{x}) \psi=\tilde{E} \psi
$$

halini alır.

\subsection{Denge-Dışı Green Fonksiyonu Yöntemi}

Burada iletim olasılığı hesabını yapacağımız sonlu farklar yöntemi bazlı denge-dışı Green fonksiyonu (SFY-DDGF) yöntemi kısaca özetlenecektir.

Sistemin gecikmeli Green fonksiyonu $\left(G^{r}\right)$ bulunmalıdır. Bunun için ilgilenilen uzay $\Delta \tilde{x}$ düzgün aralıklarla $N$ parçaya bölünür. Ölçeklendirilmiş Schrödinger dalga denklemi, sonlu farklar kesikleştirilmesi yapılarak uzayın her noktası için yazılırsa $(11 ; 15) \widetilde{U}_{n}=\widetilde{U}\left(\tilde{x}_{n}\right)$ olmak üzere: 


$$
-\tilde{t} \psi_{n-1}+\left(2 \tilde{t}+\widetilde{U}_{n}\right) \psi_{n}-\quad\left[E I-H-\Sigma_{\mathrm{L}}-\Sigma_{\mathrm{R}}\right]\{\psi\}=\{S\}
$$

$\tilde{t} \psi_{n+1}=E \psi_{n}$

elde edilir. $\tilde{t}=\frac{1}{2 \Delta \tilde{x}^{2}}$ en yakın iki komşu noktası arası etkileşme parametresidir.

Aygit ile sağ ve sol kontak etkileşmesi yani açık sınır koşulları sisteme dâhil edilirse, ölçeklendirilmiş Schrödinger dalga denklemi matris formda aşağıdaki halini alır.
$[H]$ ve $[I]$ sirasiyla $(N \times N)$ lik aygit bölgesine ait Hamiltonian ve birim matris $\{\psi\}$ dalga fonksiyonu ve $\{S\},(N \times 1)$ kolon matrisi. $\left[\Sigma_{\mathrm{L}}\right]$ ve $\left[\Sigma_{\mathrm{R}}\right]$ sirasiyla $(N \times N)$ lik sol ve sağ kontağa ait öz-enerji matrisleridir. Açık olarak Hamiltonyen matrisi

$$
\begin{aligned}
& {[H]=\left(\begin{array}{cccccccc}
2 \tilde{t}+\widetilde{U}_{1} & -\tilde{t} & 0 & & \cdots & 0 & 0 & \\
-\tilde{t} & 2 \tilde{t}+\widetilde{U}_{2} & -\tilde{t} & & \cdots & 0 & 0 & \\
0 & -\tilde{t} & 2 \tilde{t}+\widetilde{U}_{3} & & \ddots & \vdots & \vdots & \\
0 & 0 & \ddots & & \ddots & -\tilde{t} & 0 & \\
\vdots & \vdots & \ddots & -\tilde{t} & 2 \tilde{t}+\widetilde{U}_{N-1} & -\tilde{t} \\
0 & \cdots & 0 & 0 & -\tilde{t} & 2 \tilde{t}+\widetilde{U}_{N}
\end{array}\right)} \\
& {\left[\Sigma_{\mathrm{L}}\right]=\left(\begin{array}{cccc}
-\tilde{t} e^{i \tilde{k}_{L} \Delta \tilde{x}} & 0 & \ldots & 0 \\
0 & 0 & & 0 \\
\vdots & & \ddots & \vdots \\
0 & & \cdots & 0
\end{array}\right),\left[\Sigma_{\mathrm{R}}\right]=\left(\begin{array}{cccc}
0 & \cdots & & 0 \\
\vdots & \ddots & & \vdots \\
0 & \cdots & 0 & 0 \\
0 & & 0 & -\tilde{t} e^{i \tilde{k}_{R} \Delta \tilde{x}}
\end{array}\right)} \\
& \{\mathrm{S}\}=\left(\begin{array}{c}
-\tilde{t}\left(e^{i \tilde{k}_{L} \Delta \tilde{x}}-e^{-i \tilde{k}_{L} \Delta \tilde{x}}\right. \\
0 \\
\vdots \\
0
\end{array}\right) \text { şeklindedir. }
\end{aligned}
$$

Burada $\tilde{k}_{L} \quad$ ve $\tilde{k}_{R}$ sirasiyla sol ve sağ kontak düzlem dalgaların dalga vektörleridir. Matris formdaki Schrödinger dalga denkleminin Green fonksiyonu cinsinden çözümü $\{\psi\}=\left[G^{r}\right]\{S\} \quad$ şeklindedir. Burada sistemin gecikmeli Green fonksiyonu matrisi $\left(\left[G^{r}\right]\right)$ kapalı formda

$\left[G^{r}\right]=\left[(E+i \eta) I-H-\Sigma_{\mathrm{L}}-\Sigma_{\mathrm{R}}\right]^{-1}$

olarak ifade edilebilir. Denklemdeki $\eta$ sonsuz küçük pozitif bir reel sayıdır.

Sistemin Green fonksiyonu belirlendikten sonra iletim katsayıs 1

$T(\widetilde{E})=\operatorname{Tr}\left[\Gamma_{\mathrm{L}} G^{r} \Gamma_{\mathrm{R}} G^{r+}\right]$

ifadesinden hesaplanır. Burada $\Gamma_{\mathrm{L}}=\mathrm{i}\left[\Sigma_{\mathrm{L}}-\Sigma_{\mathrm{L}}^{+}\right]$ve $\Gamma_{R}=\mathrm{i}\left[\Sigma_{R}-\Sigma_{R}^{+}\right]$ile ifade edilen genişleme matrisleridir.

\section{Bulgular}

Burada TDÇB yapısının rezonans tünelleme karakteristiği incelenecektir. Sistemin etkin kütlesi yap1 boyunca $\mathrm{m}^{*}=0.067 \mathrm{~m}_{\mathrm{e}}$, dielektirik sabiti $\varepsilon_{\mathrm{r}}=$ 12,7, etkin Bohr yarıçapı $\mathrm{a}_{0}^{*}=10.1061 \mathrm{~nm}$, etkin Hartree enerjisi $E_{H}^{*}=11.2193 \mathrm{meV}$ alınmıştır. Şekil 2 de elektronların bariyerlerden geçiş olasılığının elektronun enerjisine göre değişimi görülmektedir. Elektronun enerjisi bariyer potansiyel enerjisinden küçük olduğunda kuyu içerisindeki enerji seviyesi civarında enerjiyle gelen elektronların tamamının geçebildiği gözlenmektedir. Bu olaya rezonans tünelleme olayı denir. TDÇB yapısında kuyu genişliği $\left(\mathrm{L}_{\mathrm{W}}\right) 4$ ve 8 $\mathrm{nm}$ olması durumundaki iletim katsayısının gelen elektronların enerjisine göre değişim grafiğine bakılırsa (Şekil 2(a)), daha geniş kuyuda rezonans tünellemenin olduğu enerjisinin daha düşük enerji değerinde olduğu görülür. Kuyu genişledikçe kuyu içerisindeki enerji seviyeleri daha düşük enerjilerde oluştuğundan dolayı bu durum gerçekleşmektedir. Ayrıca kuyu genişledikçe kuyu içerisinde kalan enerji seviyesi sayısı artar, böylece şekilde görüldüğü gibi $8 \mathrm{~nm}$ kuyu genişliği için ikinci bir rezonans enerjisi dolayısıyla rezonans piki oluşur. 

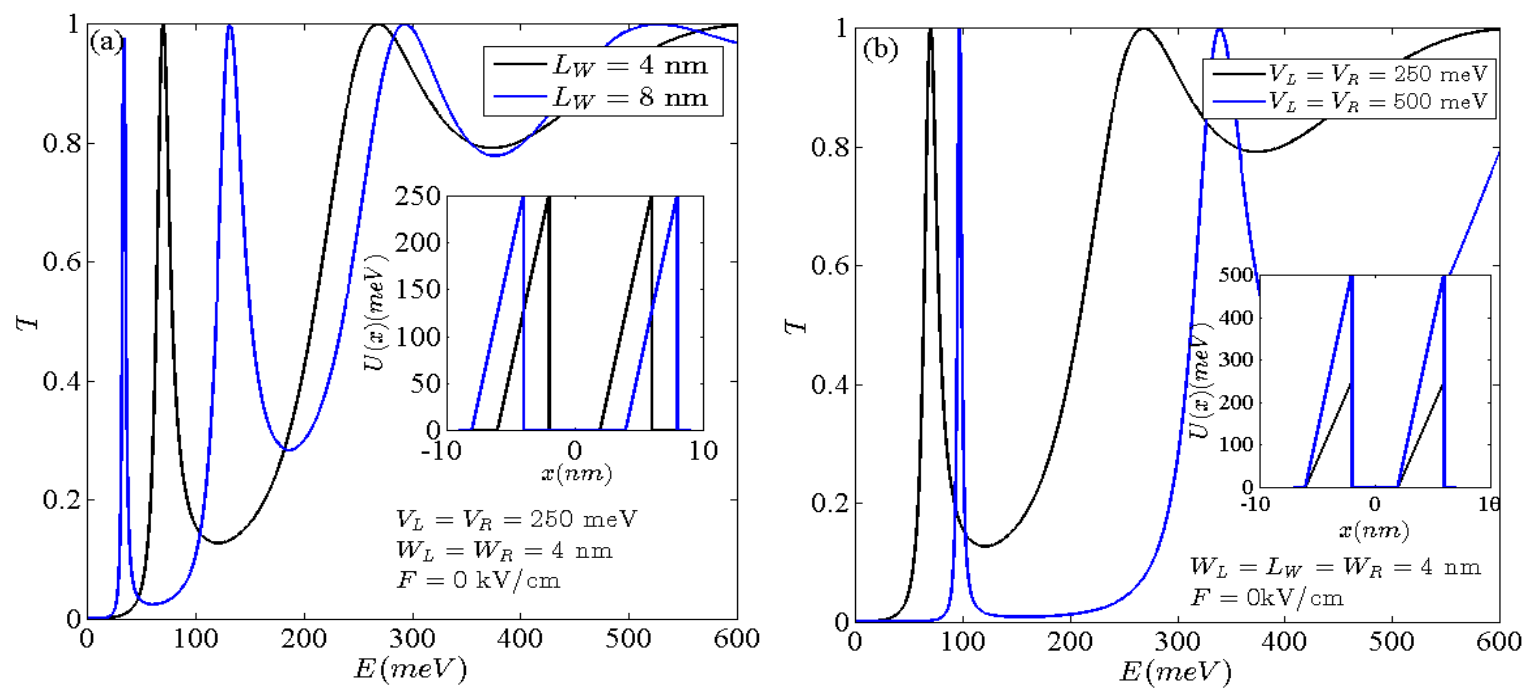

Şekil 2 İletim katsayısının gelen elektron enerjisi ile değişimi grafiği. (a) $L_{W}=4$ ve $8 \mathrm{~nm}$ için $\quad$ (b) $V_{L}=V_{R}=250$ ve $500 \mathrm{meV}$ için. Sistem parametreleri $W_{L}=W_{R}=4 \mathrm{~nm}$ ve $\mathrm{F}=0 \mathrm{kV} / \mathrm{cm}$ olarak seçilmiştir. (Dependence of the transmission coefficient with incoming electron energy (a) for well widths $L_{W}=4$ and $8 \mathrm{~nm}$ (b) for $V_{L}=V_{R}=250$ ve $500 \mathrm{meV}$. we set system parameter $\mathrm{W}_{\mathrm{L}}=\mathrm{W}_{\mathrm{R}}=4 \mathrm{~nm}$ and $\mathrm{F}=0 \mathrm{kV} / \mathrm{cm}$.)

Şekil 2(b) de ise TDÇB yapısında rezonans tünelleme olayına bariyer yüksekliğinin etkisi görülmektedir. Bariyerlerin yükseklikleri artıkça kuyudaki kuşatma daha da kuvvetlendiğinden rezonans pik genişliğinin daraldığı ve yüksek enerjiye doğru kaydığı gözlemlenmektedir. Ayrıca (seçilen yapı genişlikleri için) potansiyel yüksekliğinden rezonans pik boyunun etkilenmediği söylenmelidir.
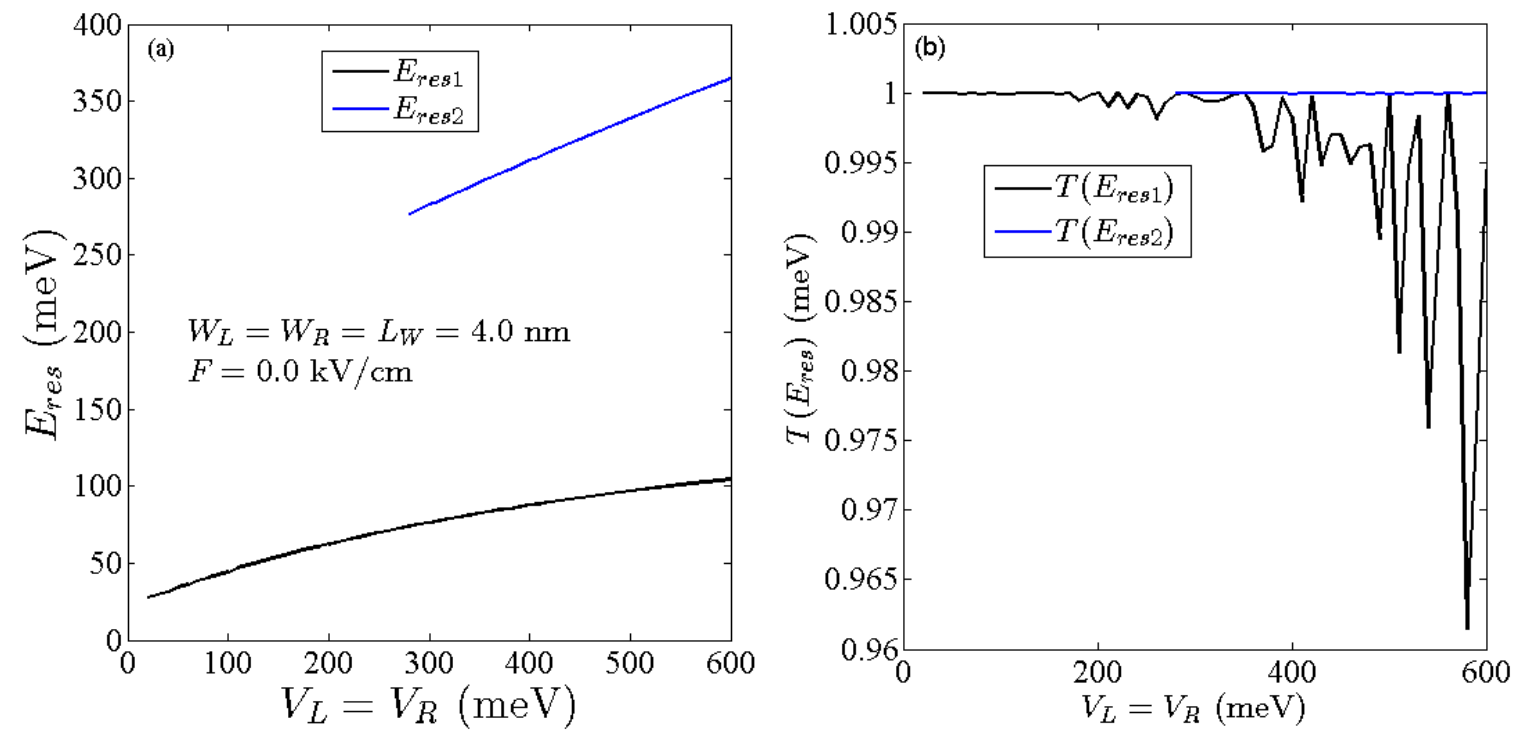

Şekil 3 (a) Rezonans enerjisinin (b) Rezonans pik genliğinin potansiyel bariyer yükseklikleri ile değişim grafiği. Sistem parametreleri $\mathrm{W}_{\mathrm{L}}=\mathrm{LW}=\mathrm{W}_{\mathrm{R}}=4 \mathrm{~nm}$ ve $\mathrm{V}_{\mathrm{L}}=\mathrm{V}_{\mathrm{R}}=250 \mathrm{meV} \mathrm{F}=0.0 \mathrm{kV} / \mathrm{cm}$ olarak seçilmiştir. (The Graph of (a) resonance energy (b) the resonance peak amplitude with respect to varying barrier heights. we fixed system parameter $\mathrm{W}_{\mathrm{L}}=\mathrm{Lw}_{\mathrm{W}}=\mathrm{W}_{\mathrm{R}}=4 \mathrm{~nm}, \mathrm{~V}_{\mathrm{L}}=\mathrm{V}_{\mathrm{R}}=250 \mathrm{meV}$ and $\mathrm{F}=0.0 \mathrm{kV} / \mathrm{cm}$.)

Şekil 3’te rezonans enerjisinin potansiyel bariyer yükseklikleri ile değişim grafiği $\mathrm{W}_{\mathrm{L}}=\mathrm{L}_{\mathrm{W}}=\mathrm{W}_{\mathrm{R}}=4 \mathrm{~nm}$ ve $\mathrm{V}_{\mathrm{L}}=\mathrm{V}_{\mathrm{R}}=250 \mathrm{meV} \mathrm{F}=0.0 \mathrm{kV} / \mathrm{cm}$ için çizilmiştir. Potansiyel bariyer yükseklikleri artıkça kuyudaki enerji seviyeleri yüksek enerjiye kaymaktadır. Böylelikle rezonans tünelleme daha yüksek enerjilerde gerçekleşmektedir. 

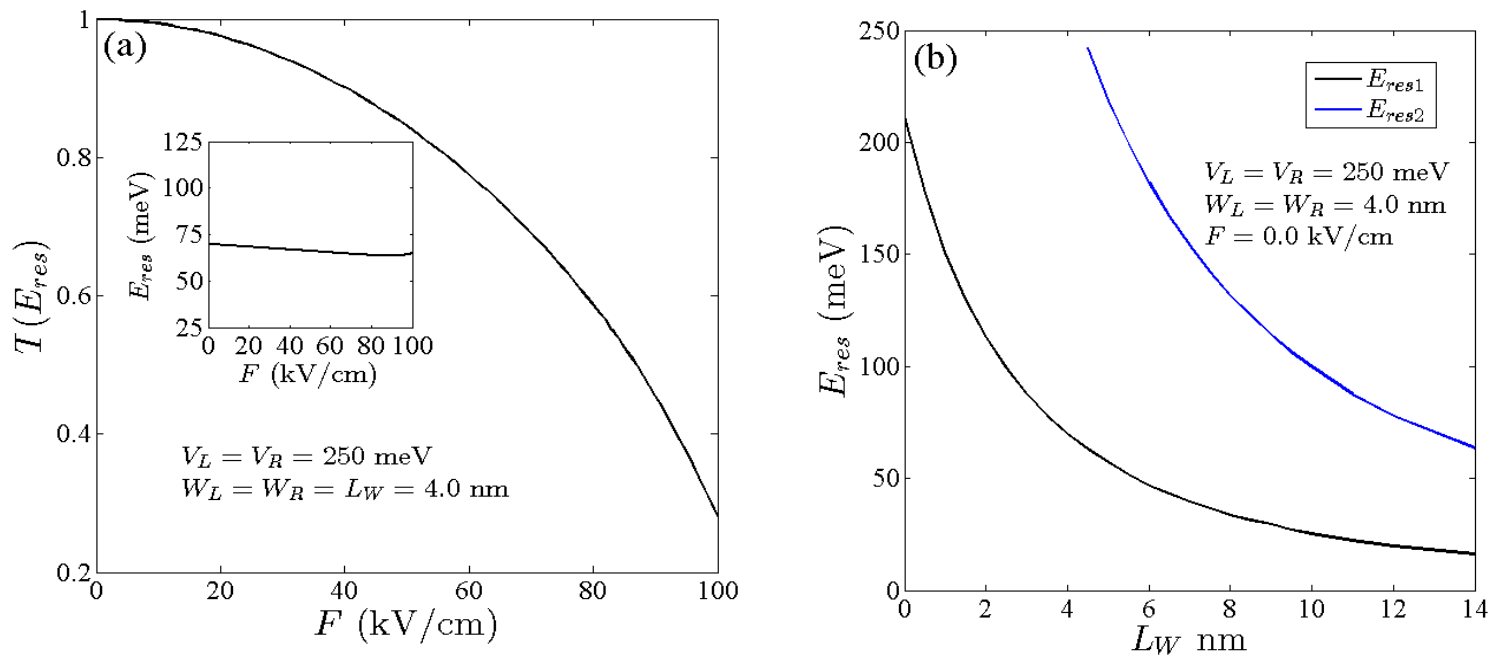

Şekil 4 (a) Rezonans pik genliğinin elektrik alan biası ile değişimi. (b) Elektrik alan biası yok iken rezonans enerjisinin kuyu genişliği ile değişim grafiği. Sistem parametreleri $W_{L}=W_{R}=4 \mathrm{~nm}$ ve $V_{L}=V_{R}=250$ meV olarak seçilmiştir. ((a) The variation of the resonance peak amplitude as a function of applied electric bias. (b) Graph of dependence of resonance energy with respect to varying width of the well without electric bias. We choose the system parameters to $\mathrm{W}_{\mathrm{L}}=\mathrm{W}_{\mathrm{R}}=4 \mathrm{~nm}$ and $\mathrm{V}_{\mathrm{L}}=\mathrm{V}_{\mathrm{R}}=250 \mathrm{meV}$ )

Şekil 4(a) da rezonans pik genliğinin elektrik alan biası ile değişimi görülmektedir. Bu şekil için kuyu ve bariyer genişlikleri $4 \mathrm{~nm}$, bariyer yükseklikleri 250 meV olarak alınmıştır. Elektrik alan biası artıkça rezonans pik genliğinin küçüldüğü görülmektedir. Şekil içerisine eklenmiş olan grafikte rezonans enerjisinin elektrik alan biası arttıkça yüksek bias değerlerine kadar düşük enerjiye kaydığı gözlemlenmektedir. Şekil 4(b) de
1. ve 2. rezonans enerjilerinin kuyu genişliği ile değişimi grafiği görülmektedir. Kuyu içerisinde, kuyu genişliği $4.5 \mathrm{~nm}$ ve üzeri değerlerinde ikinci bir (rezonans) enerji seviyesi oluşmaktadır. Kuyu genişliği arttıkça rezonans enerjisi düşük enerji değerlerine kaymaktadır. Bu değerlerden bir kaçı tablo halinde Tablo 1 de sunulmuştur. Üçüncü rezonans enerji seviyesi $\mathrm{L}_{\mathrm{W}}=9.5 \mathrm{~nm}$ den sonra oluşmaktadır.

Tablo 1. Birkaç farklı kuyu genişlikleri için kuyudaki enerji seviyeleri (Energy levels in the several different well widths)

\begin{tabular}{|l|l|l|l|}
\hline Lw $(\mathbf{n m})$ & $\mathbf{E}_{\text {res1 }}(\mathbf{m e V})\left(\mathbf{T}\left(\mathbf{E}_{\text {res1 }}\right)\right)$ & $\mathbf{E}_{\text {res2 }}(\mathbf{m e V})\left(\mathbf{T}\left(\mathbf{E}_{\text {res2 }))}\right.\right.$ & $\mathbf{E}_{\text {res3 }}(\mathbf{m e V})\left(\mathbf{T}\left(\mathbf{E}_{\text {res3 }}\right)\right)$ \\
\hline 0.0 & $211.4(1)$ & - & - \\
\hline 4.0 & $69.67(0,9997)$ & - & - \\
\hline 4.5 & $63.06(0,9964)$ & $242.0(1)$ & - \\
\hline 5.0 & $57.06(0,9976)$ & $218.6(1)$ & - \\
\hline 9.0 & $29.43(0,9666)$ & $114.1(1)$ & - \\
\hline 9.5 & $27.03(0,9493)$ & $106.3(0,999)$ & $236(1)$ \\
\hline 10 & $25.23(0,9222)$ & $99.70(0,9999)$ & $221(1)$ \\
\hline 12 & $19.82(0,9997)$ & $78.08(1)$ & $173(1)$ \\
\hline
\end{tabular}

\section{Değerlendirme ve Sonuç}

Testere dişi çift bariyer yapısında rezonans enerjisinin kuyu genişliğii, bariyer genişlikleri ve yüksekliklerine bağlılı̆̆ı sonlu farklar yöntemi bazlı denge-dışı Green fonksiyonu yöntemi kullanılarak analiz edilmiştir. Elektrik alan biası çok zayıf iken rezonans tünelleme enerjisinde gelen tüm parçacıklar tüneller yani iletim katsayısı bir (1) değerindedir. Simetri bozulduğunda veya elektrik alan şiddeti simetrik yapıda arttırıldığında iletim olasılığı azalır yani rezonans tünelleme pik şiddeti düşer. Rezonans tünelleme enerjisi kuyu genişliği arttıkça daha düşük enerji değerlerine kayarken bariyer yüksekliklerinin artmasıly daha yüksek enerjilere kaymaktadır. Bariyer yüksekliklerinin değişiminin rezonans pik şiddetine etkisi yok denecek kadar azdır. Sonuç olarak çift bariyer yapılarında rezonans tünelleme karakteristiği bu parametreler ile kontrol edilerek kullanışılı aygıt uygulamaları geliştirilebilir. 


\section{Kaynaklar}

[1]. Esaki, L. New Phenomenon in Narrow Germanium p-n Junctions. Physical Review, s. 1958, 109, 603-604.

[2]. Ferry, D.K., Goodnick, S.M. ve Bird, J. Transport in nanostructures. Cambridge : Cambridge University Press, 2009.

[3]. Levi, A.F.J. Applied Quantum Mechanics. Cambridge : Cambridge University Press, 2012.

[4]. Nutku, F. Quasi-bound levels, transmission and resonant tunneling in heterostructures with double and multi rectangular, trapezoidal, triangular barriers. J. Comput. Electron. , 2014, 13, 456.

[5]. Regan, B.C.,Surface-tension-driven nanoelectromechanical relaxation oscillator. 2005, Cilt 86, 123119, s. 86, 123119.

[6]. Wigner, E. On the quantum correction for thermodynamic equilibrium. Physical Review, 1932, 40, 749-759.

[7]. Jiang, H., Cai, W., Tsu, R. Accuracy of the Frensley inflow boundary condition for Wigner equations in simulating resonant tunneling diodes. J. Comput. Phys., 2011, 230, 2031.

[8]. Shifren, D. ve Ferry, L. Particle Monte Carlo simulation of Wigner function tunneling. Physics Letters A,, 2001, 285, 217-221.

[9]. Querlioz, D., Dollfus, P., Do, V.-N., Bournel, A., Nguyen, V. L. An improved Wigner MonteCarlo technique for the self-consistent simulation of RTDs.J. Comput. Electron., 2006, 5, 443.
[10]. Esaki, L., Tsu, R. Tunneling in a finite superlattice. Applied Physics Letters, 1973, 22, 562-564.

[11]. Datta, S. Quantum transport: atom to transistor. Cambridge: Cambridge University Press, 2005.

[12]. Mori, N., Edagawa, T., Kamakura, Y., Eaves, L. Nonequilibrium green function simulations of graphene-nanoribbon resonant-tunneling transistors. Jpn. J. Appl. Phys., 2014, 53. 04EN04.

[13]. Harrison, P. Quantum wells, wires and dots: theoretical and computational physics of semiconductor nanostructures. New York: John Wiley \& Sons, 2010.

[14]. Miyamoto, K. ve Ymamoto, H. ResonanTunneling in asymmetrical doublebarrier structures under an applied electric field. Journal of Applied Physics , 1998, 84, 311-318.

[15]. Bati, M., Sakiroglu, S. ve I. Sokmen. Electron transport in electrically biased inverse parabolic double-barrier structure. Chinise Physics B, 2016, 057307.

[16]. Ohmukai, M. Triangular double barrier resonant tunneling. Materials Science and Engineering: B, 2005,. 87-90.

[17]. Wang, H., Xu, H. ve Zhang, Y. A theoretical study of resonant tunneling characteristics in triangular double-barrier diodes. Physics Letters A, 2006, 355, 481-488.

[18]. Luo, M., Yu, G., Xia, L. Calculation of conductance for triangular multi-barrier structure in a constant electric field. Superlattices and Microstructures, 2015,. 83, 168-175. 